\title{
The molecular pathogenesis of pancreatic cancer: clarifying a complex circuitry
}

\author{
Anil K. Rustgi ${ }^{1}$ \\ Departments of Medicine and Genetics, University of Pennsylvania, Philadelphia, Pennsylvania 19104, USA
}

The preponderance of pancreatic malignancies is constituted by pancreatic ductal adenocarcinomas, abbreviated as either PDA or PDAC in the literature and used interchangeably with the generic term pancreatic cancer. Other types of pancreatic malignancies, albeit uncommon and not meant to be exhaustive, include neuroendocrine tumors, acinar cell carcinomas, lymphomas, sarcomas, serous cystadenocarcinoma, and pancreatoblastoma, among other rare entities (Hruban 2006). PDAC is a disease that has a number of clinical challenges. There are $\sim 33,000$ new cases that occur annually in the United States, with nearly the same number of deaths predominantly related to metastatic complications (Jemal et al. 2004). Overall, 5-yr survival is $<5 \%$, and this is attributable to several factors, including presentation at advanced stages of disease, thereby precluding surgical intervention, relative resistance to chemotherapy and radiation therapy, and current hurdles with other forms of therapy such as specific molecular targets, immunotherapy, and gene therapy. Meaningful therapy in the form of surgical resection is only feasible with earlystage detection, but this only applies to $<20 \%$ of patients and still results in a 5 -yr survival of $<20 \%$ (Warshaw and Fernandez-del Castillo 1992).

Through meticulous attention to morphology and nomenclature, it is now well established that PDAC has precursor lesions, termed PanIN for pancreatic intraepithelial neoplasia (Hruban et al. 2001). PanIN lesions, delineated as PanIN-1a, PanIN-1b, PanIN-2, and PanIN-3, are associated with progressive alterations in the nuclei, epithelial polarity, and architecture, and culminate in a carcinoma in situ state (PanIN-3). Nevertheless, early PanIN lesions may be found in tissues from patients with chronic pancreatitis without PDAC or from patients at autopsy with ostensibly normal pancreata. While it is widely accepted that PanIN are the preferred precursor lesions for PDAC, this does not preclude the possibility, yet to be genetically established, that other pathways may evolve into PDAC. In the context of progression to PDAC, however, each PanIN stage is associated with increasingly frequent modifications in canonical oncogenes and tumor suppressor genes, such as $\mathrm{Ki}$ -

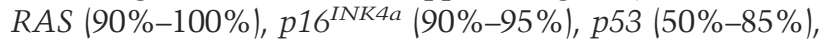
DPC4/SMAD4 (50\%), and BRCA2 (10\%) (Hruban et al. 2000; Hezel et al. 2006) This should not be construed as

${ }^{1}$ Correspondence.

E-MAIL anil2@mail.med.upenn.edu; FAX (215) 573-5412.

Article is online at http://www.genesdev.org/cgi/doi/10.1101/gad.1501106. diminishing the importance of other genes and/or pathways. Certainly, the widespread use of genomic platforms has led to the consideration of other genetic factors apart from understanding genetic-environmental interactions. In addition, there has been increasing attention to intraductal papillary mucinous neoplasms (IPMN) and mucinous cystic neoplasms (MCN), which represent other histological types of preinvasive ductal lesions that can progress to invasive carcinomas (Adsay 2005; Tanaka et al. 2006). IPMN-associated invasive carcinomas are either colloid carcinomas or PDAC (also referred to by some as invasive "tubular" carcinomas). MCN-associated invasive carcinomas are also designated as invasive tubular carcinomas or invasive adenocarcinomas (or mucinous cystoadenocarcinomas in the past). The latter tumors have focal collections of a characteristic and defining ovarian-like stroma, in which the stromal cells have "wavy" appearing nuclei and often demonstrate nuclear expression of progesterone $150 \%-$ $75 \%)$ and estrogen $(25 \%)$ receptors. In contrast to PanINassociated PDAC, those invasive cancers in association with IPMN or MCN carry a much better clinical prognosis in patients. Understanding the biological differences between the PDAC from PanIN lesions and the invasive carcinomas that arise from IPMN and MCN lesions may not only unravel important molecular insights with therapeutic implications for all three classes of invasive disease. This perspective will focus on past and current mouse models, the latter published in this particular issue of Genes \& Development, as a basis to elucidate the molecular pathogenesis of pancreatic ductal adenocarcinoma and precursor lesions. While space constraints preclude appropriate attention to translational medicine emerging from the mouse models, nevertheless implicit in this perspective is that past and current mouse models may be used as a platform for molecular diagnostics (biomarker development, imaging), prognosis (risk stratification), and targeted therapeutics.

A number of historic and landmark mouse models attempted to recapitulate human pancreatic ductal adenocarcinoma. These involved transgenic mouse models in which the acinar-specific elastase mouse promoter was fused to activated Ha-Ras, c-myc, or SV4O T antigen, resulting in either acinar cell carcinomas or mixed acinar and ductal carcinomas. A separate series of models linked expression of oncogenic stimuli to the rat insulin promoter initiated by Hanahan and colleagues (Hanahan 1985; for review, see Grippo and Sandgren 2005). The 
march to modeling PDAC then evolved to the fusion of the metallothionein promoter with transforming growth factor $\alpha(T G F-\alpha)$ in transgenic mice (Wagner et al. 1998), which resulted in acinar-ductal metaplasia and, in a small subset of mice, evidence of mixed histology tumors (specifically called mixed cystic-papillary phenotype). Interestingly, while mutations in the Ras oncogene were not detected, nuclear p53 immunoreactivity was observed, which is typically only seen with stabilizing point mutations of the protein. This provided a rationale for cross-breeding these mice with constitutively p53-null mice, resulting in a dramatic tumor development recapitulating several features of the human disease counterpart (Wagner et al. 2001). The tumors harbor biallelic deletion of Ink4a/Arf or loss of heterozygosity (LOH) of the Smad4 locus, thereby implicating that loci in addition to p53 are involved in tumor progression. The alterations in the Ink4a/Arf or Smad4 (discussed further below) proved to be prophetic and spawned different mouse experiments. Interestingly, initial crossing of the TGF- $\alpha$ mice onto the Ink4a/Arf mutant background resulted in benign serous cystadenomas, but not PDAC (Bardeesy et al. 2002).

Innovative mouse models of pancreatic ductal adenocarcinoma have gained much notoriety since 2003. The major breakthrough emerged from an approach involving the targeted endogenous expression of activated Kras $^{G 12 D}$ in presumed progenitor cells, using either $p 48$ Cre or Pdx1-Cre (Hingorani et al. 2003). These doublemutant mice recapitulated with fidelity the complete panoply of PanIN lesions. As the mice age, these PanINs also evolve spontaneously into primary, invasive PDAC with concomitant metastatic lesions. Mice harboring endogenous $\operatorname{Kras}^{G 12 D}$ expression in the pancreas have now become the workhorse of mouse modeling, opening up entirely new areas of investigation, as illustrated by the three papers in this issue of Genes \& Development by Bardeesy et al. (2006), Ijichi et al. (2006), and Pasca di Magliano et al. (2006). Interestingly, the pancreatic lesions in the $\operatorname{Kras}^{G 12 D}$ mice were confined to the ductal cell lineage, but not of the endocrine or acinar cell lineages, underscoring that the cell of origin for PanINs may reside in ductal epithelia or alternatively, undifferentiated precursor cells that default into the ductal cell lineage by virtue of the consequences of Kras mutation. More recently, it has been suggested that centroacinar cells may serve the role of progenitor cells. In the face of pancreatic specific Pten deficiency, acinar cells are replaced by proliferative ductal structures that express Pdx1 and Hes1, and some of the mice develop ductal malignancy (Stanger et al. 2005).

\section{Can Kras ${ }^{G 12 D}$ cooperate with other canonical genes, namely inactivated tumor suppressor genes, to produce PDAC?}

Kras $^{G 12 D}$ expression in combination with Ink4a/Arf deficiency in the pancreas results in earlier onset of PanIN lesions with a rapid progression to both invasive and metastatic PDAC and sarcomatoid lesions, highlighted by a significantly shorter survival period than $\operatorname{Kras}^{G 12 D}$ expression alone (Aguirre et al. 2003). This provided the first proof of the constraints imposed on pancreatic carcinogenesis by Ink4a/Arf. A natural question was to assess subsequently the functional role of p53 mutation in the context of $\operatorname{Kras}^{G 12 D}$ expression, specifically in the pancreas. Human PDAC typically show differing point mutations in one p53 allele and then $\mathrm{LOH}$ of the locus. Endogenous expression of $\operatorname{Trp} 53^{R 172 H}$ and $\mathrm{Kras}^{G 12 D}$ in the mouse pancreas demonstrated invasive PDAC with metastases to distant organs, including the liver and lungs (Hingorani et al. 2005). Furthermore, genomic instability was a salient feature of such tumors. Interestingly, no somatic mutations were noted in other key tumor suppressor genes. Thus, as with Ink4a/Arf, p53 serves to constrain the natural tendency of some PanIN lesions to progress to PDAC.

\section{Developmental biology meets cancer biology}

Mouse pancreatic development comprises two critical compartments: endocrine (islet) and exocrine (acinar and ductal). Pancreatic endocrine development requires the sustained expression of PDX1 (pancreatic and duodenal hox-1 transcription factor) (Offield et al. 1996), whereas the acinar cell lineage requires the expression of the PTF1 or p48 transcription factor (Krapp et al. 1998; Kawaguchi et al. 2002). Particularly for the well-characterized endocrine lineage, there is a hierarchy of transcription factors beyond PDX-1 expression and activation that modulate the maturation of specific cell types in the islets Pax 6, Nkx2.2, and Hnf6 for $\alpha$ cells (glucagon producing); Pdx1, Pax4, Nkx2.2, and $\beta 2 /$ NeuroD for $\beta$ cells (insulin producing); Pax 4 and Hnf6 for $\delta$ cells (somatostatin producing); and Hnf6 for pancreatic polypeptide cells. Pancreatic development or organogenesis is influenced by signals from the endoderm, mesoderm, and notochord (Kim and MacDonald 2002). In particular, Sonic hedgehog (Shh) and Indian hedgehog (Ihh) exert a negative influence on pancreatic organogenesis that is opposed by the Pdx and $\mathrm{H} 1 \mathrm{xb} 9$ transcription factors, apart from direct inhibition of Shh and Ihh by FGF and activin B from the notochord. The pancreatic progenitor stem cells require not only expression of Pdx-1 but also activated Notch and Wnt signaling.

Among the signaling pathways activated in the endogenous Kras $^{G 12 D}$ expression in the mouse pancreas were Hedgehog and Notch, suggesting a re-emergence of critical pancreatic developmental networks during carcinogenesis (Hingorani et al. 2003). However, ectopic expression of Shh in the pancreas with the $\mathrm{Pdx}-1$ promoter resulted in abnormal tubular structures, but not PDAC (Thayer et al. 2003). That being said, the lesions still demonstrated evidence of KRas mutations, along with HER-2/neu overexpression. Human PDAC reveal evidence of activated hedgehog signaling and pharmacological inhibition of Shh in tumors of xenotransplantation models are compelling proof of the role of Shh in pancreatic carcinogenesis (Berman et al. 2003; Thayer et al. 2003). A logical outcome of these findings was to cross 
the Kras ${ }^{G 12 D}$ mice with ligands or effectors of the Shh pathway. Indeed, such is the case with the approach by Pasca di Magliano et al. (2006) in this issue of Genes \& Development. Here, using overexpression of Gli2, a transcriptional activator and regulator of $\mathrm{HH}$ signaling during development, Hebrok and coworkers (Pasca di Magliano et al. 2006) generated Pdx1-cre/CLEG2/Kras ${ }^{G 12 D}$ mice, where a dominant active form of Gli-2 (CLEG2) is expressed conditionally in the pancreas upon Cre-mediated recombination in concert with Kras $^{G 12 D}$ expression. Interestingly, while the mice develop PanIN lesions, they do not progress to PDAC lesions. Rather, they display largely undifferentiated, anaplastic tumors. Of note, the Pdx1-cre/CLEG2 mice alone do not reveal evidence of PanIN lesions but do develop undifferentiated tumors. Thus, it would appear that activated Kras and Shh signaling cooperate in an unexpected manner to preserve the appearance of PanIN lesions, but "default" into a type of tumor that is undifferentiated, and perhaps resembles epithelial-mesenchymal transition (EMT), in light of the expression of mesenchymal markers such as vimentin and S-100A4, and of note. EMT is also observed in the combination of $\mathrm{Kras}^{\mathrm{G12V}}$ and $p 53^{V 143 \mathrm{~A}}$ in pancreatic cancer (Deramaudt et al. 2006) Hebrok and coworkers (Pasca di Magliano et al. 2006) surmise that Kras signaling can stimulate $\mathrm{HH}$ signaling via activation of ligand expression (modeled in Fig. 1).

Although the $\mathrm{HH}$ pathway has several negative feedback loops (e.g., Patched and Hhip proteins) that serve to constrain $\mathrm{HH}$ signaling, it is likely that Gli2-mediated transcription cannot be negatively regulated and is unencumbered with resulting undifferentiated tumors. Cooperation between $\mathrm{SHH}$ and mitogen-activated protein kinase (MAPK) in a cell-autonomous fashion has been shown to be needed for the induction of neocortical precursors. Cooperation is manifest further between $\mathrm{SHH}$

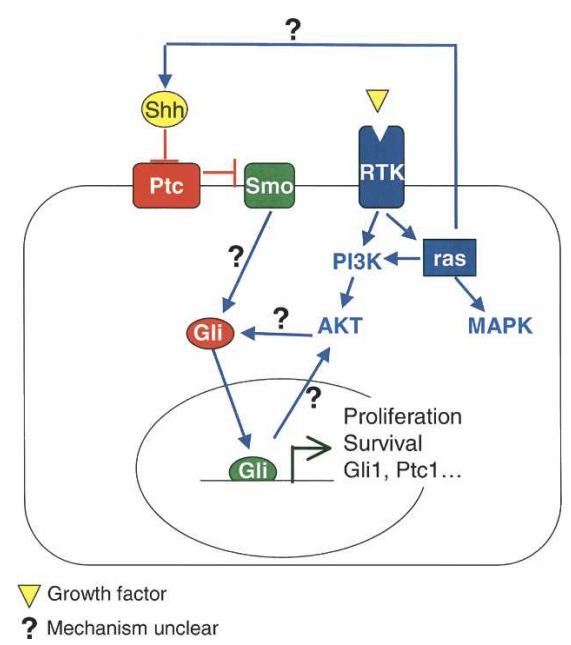

Figure 1. Cooperativity between Shh and Ras pathways. Potential areas of interaction include the notion that Shh expression is downstream from Ras in the presence of p53 mutation in the pancreas, a reciprocal activation between AKT and Gli2 in fibroblasts, and activated Kras in turn activates Shh with Gli2, activating AKT in the pancreas. and RAS in the spatial positioning of cardiac progenitor cells. The current findings, in the face of observations in nonpancreatic progenitor cells, underscore the potential cooperation of normal levels of Kras and $\mathrm{SHH}$ pathways in maintaining pancreatic progenitor cells. However, in the event of sustained activation of both pathways, such pancreatic progenitor cells are prone to nefarious malignant transformation that may evolve beyond PDAC or bypass PDAC to a highly undifferentiated, anaplastic state. Arresting these mice in a stage of differentiated tumors may be challenging in that Gli2 activation will likely escape known pharmacological inhibition of Shh.

Waiting on the sidelines and signaling to enter the pancreatic circuit is Notch, also a key determinant of pancreatic development. We know that expression of $\mathrm{Kras}^{\mathrm{G} 12 \mathrm{D}}$ in the pancreas induces Notch signaling, as illustrated by Hes-1 immunoreactivity (Miyamoto et al. 2003). Notch signaling is activated in mouse and human preneoplastic and neoplastic lesions of the pancreas. Pasca di Magliano et al. (2006) posit that $\mathrm{HH}$ signaling may also activate components of Notch signaling and Notch target genes. Taken together, natural experiments involve interrogating whether endogenous Kras ${ }^{\mathrm{G} 12 \mathrm{D}}$ expression in the pancreas may cooperate with Notch signaling in mice and also with the aforementioned genetic alterations.

The TGF $\beta$ signaling pathway acts as molecular switch in the pancreatic cancer circuitry

TGF $\beta$ has protean effects on cellular behavior and homeostasis, including proliferation and differentiation (Derynck et al. 2001; Siegel and Massague 2003). Whereas it is often viewed as a suppressor of normal cellular proliferation, TGF $\beta$ signaling may enable tumor cell growth advantage through effects mediated upon extracellular matrix deposition, promoting EMT, fostering alterations on the immune system, and angiogenesis. TGF $\beta$ itself is known to be overexpressed in human PDAC. Individual components of the TGF $\beta$ signaling pathway are deranged in human PDAC, including TGFßIIR (Venkatasubbarao et al. 2000) and Smad4 (Hahn et al. 1996; Hansel et al. 2003). TGFßIIR expression is decreased and Smad 4 alteration may occur by intragenic mutation or deletion. Ijichi et al. (2006) used the Ptf1acre mice to cross with the Tgfbr2 flox/flox mice alone or in combination with the Kras ${ }^{G 12 D}$ mice. Interestingly, the pancreas selective deletion of Tgfbr2 did not result in any discernible pancreas lesions. Once again, endogenous expression of $\mathrm{Kras}^{\mathrm{G12D}}$ was required to initiate preinvasive disease. The combination of pancreas selective $\operatorname{Kras}^{G 12 D}$ expression with Tgfbr2 knockout resulted in the full spectrum of PanIN lesions and well-differentiated PDAC with full penetrance. Median survival was nearly 2 mo, approximately the same as the combination of $\mathrm{Kras}^{\mathrm{G12D}}$ and combined INK4a/Arf deletion (Aguirre et al. 2003) shorter than what was noted in the combination of $\operatorname{Trp} 53^{R 172 H}$ and $\operatorname{Kras}^{G 12 D}$ (Hingorani et al. 2005). The latter mice, as noted previously, also have a greater spectrum of metastatic lesions, perhaps reflecting longer survival compared with the mice investigated 
by Moses and coworkers (Ijichi et al. 2006). Moses and colleagues also demonstrate convincingly that haploinsufficiency of TGF $\beta$ signaling also yields PDAC. Not only is there faithful recapitulation of human PDAC, reminiscent of the model by Hingorani et al. (2005), but also new opportunities may now be available for the blockade of TGF $\beta$ and signaling components in an attempt to retard PDAC progression.

In a similar vein, Bardeesy et al. (2006) report that Smad4 is not needed for normal pancreatic development. The combination of Smad4 deletion in the pancreatic epithelium with Kras $^{G 12 D}$ expression resulted in perhaps an unexpected, but interesting, finding of IPMN with progression to invasive PDAC at low penetrance. Furthermore, Bardeesy et al. (2006) demonstrated that the combination of Smad4 deficiency, Kras ${ }^{G 12 D}$, and Ink4a/ Arf heterozygosity accelerates the development of PDAC. Taken together, these two studies underscore a critical role for TGF $\beta$ signaling through Smad4-dependent and Smad-4 independent modes of action, and in either context, there may be concomitant cooperation with activated Ras (Fig. 2). There are clear therapeutic implications in identifying which subset of PDAC have intact or altered Smad4 or normal or down-regulated TGFBIIR as a basis for using specific inhibitors for the receptor or its downstream effectors.

Hingorani and colleagues (pers comm.; K. Izeradjene, C. Combs, M. Best, A. Gopinathan, A. Wagner, W.M. Grady, C.-X. Deng, R.H. Hruban, N.V. Adsay, D. Tuve-

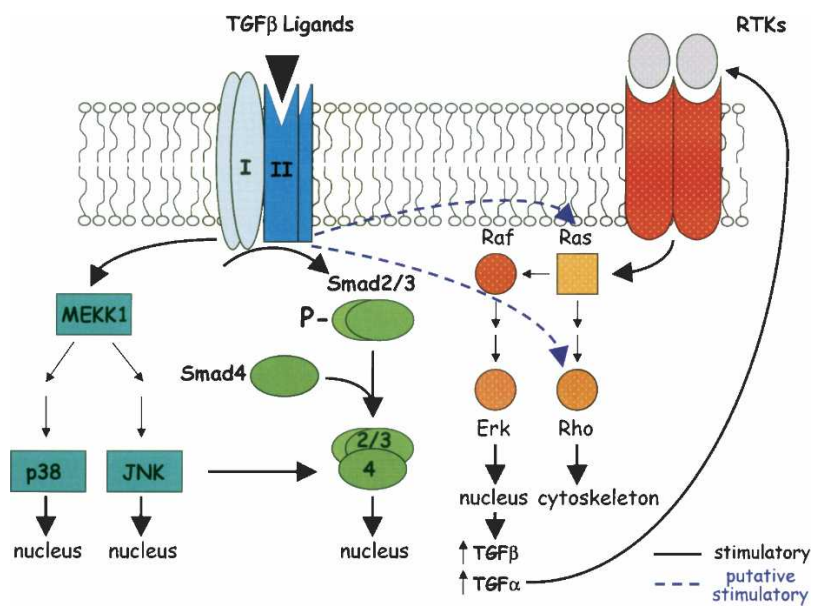

Figure 2. Cooperativity between the TGF $\beta$ and Ras pathways. Several points of direct and indirect interaction exist between these two central signaling pathways, most of which are synergistic. Ligand binding to type II receptors results in activation of MAPK pathways through stimulation of RAS and MEKK1 and, ultimately, alterations in nuclear transcription that further promote TGF $\beta$ effects. In addition, some of these pathway members can directly interact with TGF $\beta$ effectors, as seen, for example, with phosphorylation of Smad3 by JNK. MAPK activation can also lead to increased levels of TGF $\beta$ and TGF $\alpha$, each of which represent positive feedback loops in their respective cascades. Activation of the Rac and Rho GTPases, as well as p38, contributes to membrane ruffling and EMT. The ultimate cellular consequences of TGF $\beta$ stimulation depend on the balance between these various inputs in a given cellular context. son, and S.R. Hingorani, in prep.) have also examined the effects of disrupted TGF $\beta$ signaling in the context of pancreatic Kras ${ }^{G 12 D}$ expression. Although they too find that cystic neoplasms develop, the resultant lesions appear to most closely resemble MCNs. The reasons for the differences in these two genetically similar models are unclear, although they may have to do with strain differences and/or the specifics of the genetically engineered Dpc4 mutant alleles. When these lesions progress to invasive carcinomas, they do so by mutation of $p 53$ or $p 16$. These mice also live longer than each of the compound mutant animals described above.

\section{To be PanIn or not to be PanIN}

The current studies underscore that activated Ras is needed for the initiation and development of PanIN. This phenomenon cannot be supplanted by activated Shh signaling, as manifest at least by Gli2 overexpression, or disruption of Tgfbr2. However, Smad4 deficiency triggers a "conversion" to cystic neoplasms even in the face of $\mathrm{Kras}^{G 12 D}$ expression. What about frank tumor development in combination with $\operatorname{Kras}^{G 12 D}$ expression? Tgfbr2 deletion results in faithful recapitulation of human PDAC, in concert with what has been also observed with p53 deletion in the pancreas. Shh signaling activation yields undifferentiated, anaplastic tumors. Smad4 deficiency does not promote an accelerated course to tumor development, except in concert with Ink4a/Arf heterozygosity. Collectively, these reports support the notion that Shh signaling and TGF $\beta$ signaling cooperate with Ras. In the former case, this may be needed to help maintain the pool of pancreatic progenitor cells, which is easily susceptible to malignant transformation with activation of both pathways. TGF $\beta$ signaling, at least in part through Smad4, but also independent of Smad4, can block progression of PanIN to PDAC. However, specific genetic deletion of Smad4 triggers the development of cystic neoplasms, which carries a much more favorable prognosis in patients than PanIN-related PDAC. This may have important implications in deciphering the different molecular signatures between intact Smad4 and disrupted Smad4 signaling and their accompanying different precursor lesions and should encourage the investigaton of strategies inhibiting specific components of the TGF $\beta$ signaling pathway in both preclinical models and human clinical trials.

\section{Acknowledgments}

I apologize for the inability to contain all references on this topic in this perspective. This work was supported by NIH R01 DK60694.

\section{References}

Adsay, N.V. 2005. Pathological classification of cystic neoplasms of the pancreas. In Pancreatic cancer (eds. D.D. Von Hoff et al.), pp. 716-756. Jones and Bartlett, Sudbury, MA.

Aguirre, A.J., Bardeesy, N., Sinha, M., Lopez, L., Tuveson, D.A., Horner, J., Redston, M.S., and DePinho, R.A. 2003. Activated Kras and Ink4a/Arf deficiency cooperate to produce metastatic pancreatic ductal adenocarcinoma. Genes \& Dev. 17: 3112-3126. 
Bardeesy, N., Morgan, J., Sinha, M., Signoretti, S., Srivastava, S., Loda, M., Merlino, G., and DePinho, R.A. 2002. Obligate roles for $\mathrm{p} 16^{\text {Ink4a }}$ and $\mathrm{p} 19^{\text {Arf }}-\mathrm{p} 53$ in the suppression of murine pancreatic neoplasia. Mol. Cell. Biol. 22: 635-643.

Bardeesy, N., Cheng, K.-h., Berger, J.H., Chu, G.C., Pahler, J., Olson, P., Hezel, A.F., Horner, J., Lauwers, G.Y., Hanahan, D., et al. 2006. Smad4 is dispensable for normal pancreas development yet critical in progression and tumor biology of pancreas cancer. Genes \& Dev. (this issue).

Berman, D.M., Karhadkar, S.S., Maitra, A., Montes De Oca, R., Gerstenblith, M.R., Briggs, K., Parker, A.R., Shimada, Y., Eshleman, J.R., Watkins, D.N., et al. 2003. Widespread requirement for Hedgehog ligand stimulation in growth of digestive tract tumours. Nature 425: 846-851.

Deramaudt, T.B., Takaoka, M., Upadhyay, R., Bowser, M.J., Porter, J., Lee, A., Rhoades, B., Johnstone, C.N., Weissleder, R., Hingorani, S.R., et al. 2006. N-cadherin and keratinocyte growth factor receptor mediate the functional interplay between Ki-RAS ${ }^{\mathrm{G} 12 \mathrm{~V}}$ and $\mathrm{p} 53^{\mathrm{V} 143 \mathrm{~A}}$ in promoting pancreatic cell migration, invasion, and tissue architecture disruption. Mol. Cell. Biol. 26: 4185-4200.

Derynck, R., Akhurst, R.J., and Balmain, A. 2001. TGF- $\beta$ signaling in tumor suppression and cancer progression. Nat. Genet. 29: 117-129.

Grippo, P.J. and Sandgren, E.P. 2005. Modeling pancreatic cancer in animals to address specific hypotheses. Methods Mol. Med. 103: 217-243.

Hahn, S.A., Schutte, M., Hoque, A.T., Moskaluk, C.A., da Costa, L.T., Rozenblum, E., Weinstein, C.L., Fischer, A., Yeo, C.J., Hruban, R.H., et al. 1996. DPC4, a candidate tumor suppressor gene at human chromosome 18q21.1. Science 271: 350-353.

Hanahan, D. 1985. Heritable formation of pancreatic $\beta$-cell tumours in transgenic mice expressing recombinant insulin/ simian virus 40 oncogenes. Nature 315: 115-122.

Hansel, D.E., Kern, S.E., and Hruban, R.H. 2003. Molecular pathogenesis of pancreatic cancer. Annu. Rev. Genomics Hum. Genet. 4: 237-256.

Hezel, A.F., Kimmelman, A.C., Stanger, B.Z., Bardeesy, N., and Depinho, R.A. 2006. Genetics and biology of pancreatic ductal adenocarcinoma. Genes \& Dev. 20: 1218-1249.

Hingorani, S.R., Petricoin, E.F., Maitra, A., Rajapakse, V., King, C., Jacobetz, M.A., Ross, S., Conrads, T.P., Veenstra, T.D., Hitt, B.A., et al. 2003. Preinvasive and invasive ductal pancreatic cancer and its early detection in the mouse. Cancer Cell 4: 437-450.

Hingorani, S.R., Wang, L., Multani, A.S., Combs, C., Deramaudt, T.B., Hruban, R.H., Rustgi, A.K., Chang, S., and Tuveson, D.A. 2005. Trp53 ${ }^{\mathrm{R} 172 \mathrm{H}}$ and $\mathrm{Kras}^{\mathrm{G} 12 \mathrm{D}}$ cooperate to promote chromosomal instability and widely metastatic pancreatic ductal adenocarcinoma in mice. Cancer Cell 7: 469-483.

Hruban, R.H. 2006. Tumors of the pancreas. In Atlas of tumor pathology (eds. R.H. Hruban et al.). Armed Forces Institute of Pathology, Washington, DC.

Hruban, R.H., Wilentz, R.E., and Kern, S.E. 2000. Genetic progression in the pancreatic ducts. Am. J. Pathol. 156: 1821-1825.

Hruban, R.H., Adsay, N.V., Albores-Saavedra, J., Compton, C., Garrett, E.S., Goodman, S.N., Kern, S.E., Klimstra, D.S., Kloppel, G., Longnecker, D.S., et al. 2001. Pancreatic intraepithelial neoplasia: A new nomenclature and classification system for pancreatic duct lesions. Am. J. Surg. Pathol. 25: 579-586.

Ijichi, H., Chytil, A., Gorska, A.E., Aakre, M.E., Fujitani, Y., Fujitani, S., Wright, C.V.E., and Moses, H.L. 2006. Aggressive pancreatic ductal adenocarcinoma in mice caused by pancreas-specific blockade of transforming growth factor- $\beta$ signaling in cooperation with active Kras expression. Genes \& Dev. (this issue).

Jemal, A., Tiwari, R.C., Murray, T., Ghafoor, A., Samuels, A., Ward, E., Feuer, E.J., and Thun, M.J. 2004. Cancer statistics, 2004. CA Cancer J. Clin. 54: 8-29.

Kawaguchi, Y., Cooper, B., Gannon, M., Ray, M., MacDonald, R.J., and Wright, C.V. 2002. The role of the transcriptional regulator Ptfla in converting intestinal to pancreatic progenitors. Nat. Genet. 32: 128-134.

Kim, S.K. and MacDonald, R.J. 2002. Signaling and transcriptional control of pancreatic organogenesis. Curr. Opin. Genet. Dev. 12: 540-547.

Krapp, A., Knofler, M., Ledermann, B., Burki, K., Berney, C., Zoerkler, N., Hagenbuchle, O., and Wellauer, P.K. 1998. The bHLH protein PTF1-p48 is essential for the formation of the exocrine and the correct spatial organization of the endocrine pancreas. Genes \& Dev. 12: 3752-3763.

Miyamoto, Y., Maitra, A., Ghosh, B., Zechner, U., Argani, P., Iacobuzio-Donahue, C.A., Sriuranpong, V., Iso, T., Meszoely, I.M., Wolfe, M.S., et al. 2003. Notch mediates TGF $\alpha$-induced changes in epithelial differentiation during pancreatic tumorigenesis. Cancer Cell 3: 565-576.

Offield, M.F., Jetton, T.L., Labosky, P.A., Ray, M., Stein, R.W., Magnuson, M.A., Hogan, B.L., and Wright, C.V. 1996. PDX-1 is required for pancreatic outgrowth and differentiation of the rostral duodenum. Development 122: 983-995.

Pasca di Magliano, M., Sekine, S., Ermilov, A., Ferris, J., Dlugosz, A.A., and Hebrok, M. 2006. Hedgehog/Ras interactions regulate early stages of pancreatic cancer. Genes \& Dev. (this issue).

Siegel, P.M. and Massague, J. 2003. Cytostatic and apoptotic actions of TGF- $\beta$ in homeostasis and cancer. Nat. Rev. Cancer 3: $807-821$.

Stanger, B.Z., Stiles, B., Lauwers, G.Y., Bardeesy, N., Mendoza, M., Wang, Y., Greenwood, A., Cheng, K.H., McLaughlin, M., Brown, D., et al. 2005. Pten constrains centroacinar cell expansion and malignant transformation in the pancreas. Cancer Cell 8: 185-195.

Tanaka, M., Chari, S., Adsay, V., Fernandez-del Castillo, C., Falconi, M., Shimizu, M., Yamaguchi, K., Yamao, K., and Matsuno, S. 2006. International consensus guidelines for management of intraductal papillary mucinous neoplasms and mucinous cystic neoplasms of the pancreas. Pancreatol. 6: $17-32$.

Thayer, S.P., di Magliano, M.P., Heiser, P.W., Nielsen, C.M., Roberts, D.J., Lauwers, G.Y., Qi, Y.P., Gysin, S., Fernandezdel Castillo, C., Yajnik, V., et al. 2003. Hedgehog is an early and late mediator of pancreatic cancer tumorigenesis. $\mathrm{Na}$ ture 425: 851-856.

Venkatasubbarao, K., Ahmed, M.M., Mohiuddin, M., Swiderski, C., Lee, E., Gower Jr., W.R., Salhab, K.F., McGrath, P., Strodel, W., and Freeman, J.W. 2000. Differential expression of transforming growth factor $\beta$ receptors in human pancreatic adenocarcinoma. Anticancer Res. 20: 43-51.

Wagner, M., Luhrs, H., Kloppel, G., Adler, G., and Schmid, R.M. 1998. Malignant transformation of duct-like cells originating from acini in transforming growth factor transgenic mice. Gastroenterology 115: 1254-1262.

Wagner, M., Greten, F.R., Weber, C.K., Koschnick, S., Mattfeldt, T., Deppert, W., Kern, H., Adler, G., and Schmid, R.M. 2001. A murine tumor progression model for pancreatic cancer recapitulating the genetic alterations of the human disease. Genes \& Dev. 15: 286-293.

Warshaw, A.L. and Fernandez-del Castillo, C. 1992. Pancreatic carcinoma. N. Engl. J. Med. 326: 455-465. 


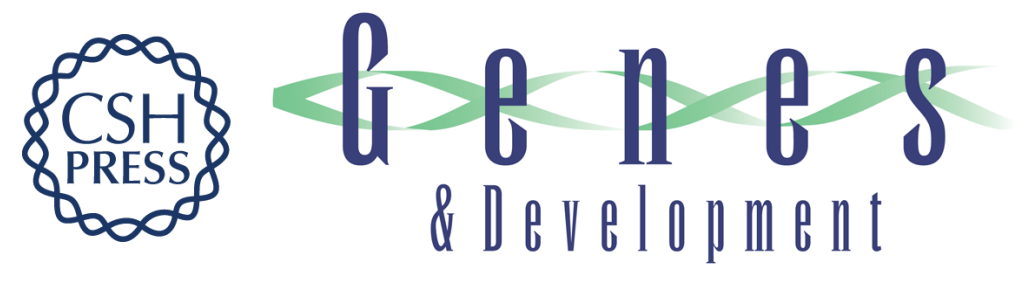

\section{The molecular pathogenesis of pancreatic cancer: clarifying a complex circuitry}

Anil K. Rustgi

Genes Dev. 2006, 20:

Access the most recent version at doi:10.1101/gad.1501106

\section{Related Content}

Smad4 is dispensable for normal pancreas development yet critical in progression and tumor biology of pancreas cancer

Nabeel Bardeesy, Kuang-hung Cheng, Justin H. Berger, et al.

Genes Dev. November , 2006 20: 3130-3146

References This article cites 28 articles, 8 of which can be accessed free at:

http://genesdev.cshlp.org/content/20/22/3049.full.html\#ref-list-1

Articles cited in:

http://genesdev.cshlp.org/content/20/22/3049.full.html\#related-urls

\section{License}

Email Alerting

Service

Receive free email alerts when new articles cite this article - sign up in the box at the top right corner of the article or click here.

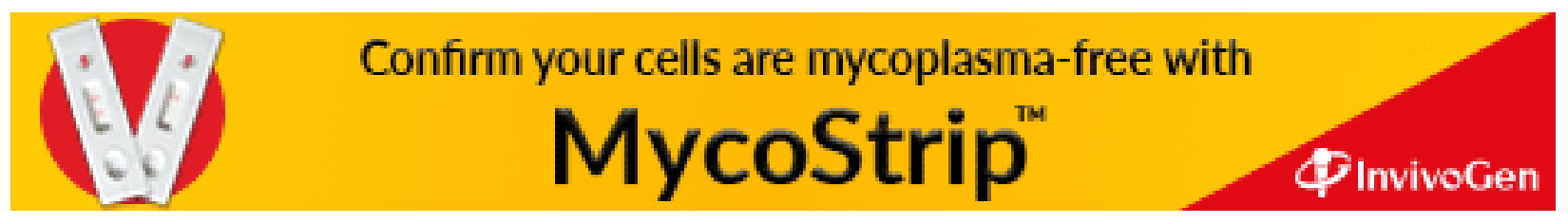

